\title{
Intussuscepção intestinal em adultos jovens - relato de caso e revisão de literatura
}

\author{
Intestinal intussusception in young adults - literature review
}

Bernardo Nogueira Batista ${ }^{1}$; Linda Ferreira Maximiano TCBC-SP²

\section{R E S U M O}

\begin{abstract}
Embora sejam a principal causa de obstrução intestinal na população pediátrica, intussuscepções intestinais são eventos raros em adultos e quando acontecem, têm características clínicas diferentes dos seus equivalentes em crianças. O objetivo desse trabalho é de apresentar um caso de um jovem do sexo masculino, de 16 anos, previamente hígido, que procurou o serviço de emergência do Hospital Universitário da Universidade de São Paulo com um quadro de intussuscepção intestinal como primeira manifestação de um linfoma não-Hodgkin difuso de células B de alto grau, tipo Burkitt. Foi realizada uma revisão da literatura pertinente, e aspectos relevantes do caso são discutidos à luz dessas informações.
\end{abstract}

Descritores: Intussuscepção. Abdome agudo. Linfoma. Linfoma de Burkitt.

\section{INTRODUÇÃO}

A intussuscepção é uma causa menos freqüente de abdome obstrutivo na população geral, e nos adultos, tem incidência, localização e causa diversa da população infantil. Principal causa de suboclusão intestinal na faixa pré-escolar, tem como localização preferencial a transição ileocecal. Tomando como mote do estudo um quadro suboclusivo em um adolescente de 16 anos, abordamos a intussuscepção como um quadro de exclusão entre as obstruções de intestino delgado na população adulta e, igualmente pouco freqüente, a ocorrência de neoplasia de intestino delgado. A presença de uma alteração morfológica, tumoral ou não, torna fundamental a extirpação cirúrgica da mesma, mesmo após resolução espontânea, ou com o auxílio de métodos endoscópicos. A identificação da intussuscepção nessa população, por si, já tem indicação cirúrgica de ressecção segmentar, sem redução intra-operatória.

\section{RELATO DE CASO}

Paciente de 16 anos, sexo masculino, pardo, procurou o Pronto Socorro do Hospital Universitário da Universidade de São Paulo com queixa de dor abdominal há uma semana. Duas semanas antes da consulta, tinha apresentado quadro de dor abdominal difusa, em cólica, de grande intensidade, que cedeu ao anti-espasmódico aplicado em outro serviço. Passou uma semana apresentando dores leves, esporádicas. Na semana anterior, a dor voltou e como não apresentou melhora com a mesma medicação utilizada no primeiro episódio, buscou atendimento médico. Referia dois episódios de vômitos no dia da consulta, sem sangue associado a diarréia liquida. Negava febre, negava história de dor abdominal anterior à relacionada a esta consulta, negava comorbidades. Ao exame físico abdominal apresentava dor à palpação profunda de fossa ilíaca direita, dor a descompressão brusca abdominal, sem visceromegalias, sem massas ou tumorações palpáveis. Uma peculiaridade do exame era que à palpação da fossa ilíaca direita não se palpavam alças nessa região.

Exames laboratoriais e radiografias abdominais não mostraram alterações. A ultra-sonografia abdominal mostrou em flanco e hipocôndrio direito uma imagem compatível com alça intestinal em fundo cego, irregularmente espessada, com imagem sugestiva de coleção adjacente a sua extremidade de $10 \mathrm{ml}$, podendo corresponder a processo inflamatório apendicular, não podendo ser descartado, mais remotamente, quadro de invaginação intestinal (imagem similar a "alça dentro de alça"). Foi então solicitada uma colonoscopia para esclarecimento diagnóstico. Notou-se então grande projeção do íleo terminal através da válvula ileocecal, que foi reduzida com o colonoscópio. Após a redução foi observado um nódulo, com mucosa levemente isquêmica e tendo $5 \mathrm{~cm}$ de diâmetro, que poderia corresponder a uma lesão funcionando como cabeça da intussuscepção ou apenas ao edema da parede ileal em sofrimento (Figura 1).

Foi agendada uma nova colonoscopia para reavaliar a lesão uma semana após a redução e determinar a conduta cirúrgica, mas antes da realização do novo

Trabalho realizado no Hospital Universitário da Universidade de São Paulo- São Paulo - SP-BR.

1. Residente de Cirurgia da Faculdade de Medicina da Universidade de São Paulo. - São Paulo - SP-BR. 2. Cirurgiã Geral da Divisão de Cirurgia do Hospital Universitário da Universidade de São Paulo - São Paulo - SP-BR. 
exame o paciente procurou novamente o pronto-socorro com recorrência do quadro clínico. O paciente foi submetido a uma laparotomia exploradora mediana na qual se observou uma invaginação de aproximadamente $10 \mathrm{~cm}$ de íleo terminal para dentro do cólon direito. A conduta cirúrgica foi colectomia parcial direita com ressecção de íleo distal em seus $20 \mathrm{~cm}$ mais distais, com uma enterocoloanastomose mecânica látero-lateral. O paciente evoluiu bem, recebendo alta no $3^{\circ}$ pós-operatório.

O estudo anatomopatológico da peça cirúrgica mostrou uma neoplasia elevada, com esboço polipóide séssil, localizada na região distal do íleo terminal, medindo $4,5 \times 3 \times 3 \mathrm{~cm}$, infiltrando até a subserosa, com margens cirúrgicas livres. Dos 18 linfonodos dissecados do tecido adiposo mesentérico, 6 apresentavam linfoma. O perfil imunohistoquímico da peça se mostrou compatível com linfoma de Burkitt, quando correlacionado ao aspecto histológico. (ki-67 positivo, Tdt inconclusivo, CD-99 negativo, CD-10 positivo, Bcl-2 negativo e EBV negativo). O paciente foi encaminhado ao serviço de oncologia referenciado para quimioterapia.

\section{REVISÃO DA LITERATURA}

\section{Intussuscepção ${ }^{1-8}$}

A intussuscepção intestinal é uma doença da população pediátrica e a maioria dos eventos sintomáticos se deve à hiperplasia do tecido linfóide das placas de Peyer. Entre as crianças, trata-se da principal causa de obstrução intestinal. Em adultos, trata-se de manifestação rara e, quando ocorre, apresenta características clínicas consideravelmente diferentes. Na presença de peritonismo normal, a ocorrência de uma tumoração intraluminal serve como elemento de tração do restante da alça que a precede, e este é o mecanismo de ação pelo qual a intussuscepção ocorre. Um estudo de 745 casos de intussuscepção intestinal diagnosticados cirurgicamente mostrou que $52 \%$ se originaram no intestino delgado, sendo 39\% enteroentéricas, e 13\% ileocólicas ${ }^{1}$. A maioria das intussuscepções em adultos diagnosticadas durante cirurgias são secundárias a lesões estruturais. Enquanto na população pediátrica 95\% dos eventos são idiopáticos² , nos adultos a maioria dos casos está relacionada a presença de uma massa funcionando com "cabeça de intussuscepção" ${ }^{3}$. Em séries recentes, apenas 16\% das intussuscepções de intestino delgado foram idiopáticas. Os neoplasmas são responsáveis por dois terços dos eventos na maioria das séries cirúrgicas, $60 \%$ deles lesões malignas. A maior porcentagem de lesões malignas encontra-se, como é de se esperar, em lesões colônicas. No intestino delgado, $57 \%$ das intussuscepções são secundárias a tumores, dos quais apenas 30\% são malignos. Desses, metástases de melanomas são a causa mais comum. As causas nãoneoplásicas mais comuns em intussuscepções de intestino delgado são as alterações morfológicas, adesões pós-cirúrgicas, e divertículos de Meckel $^{4}$.
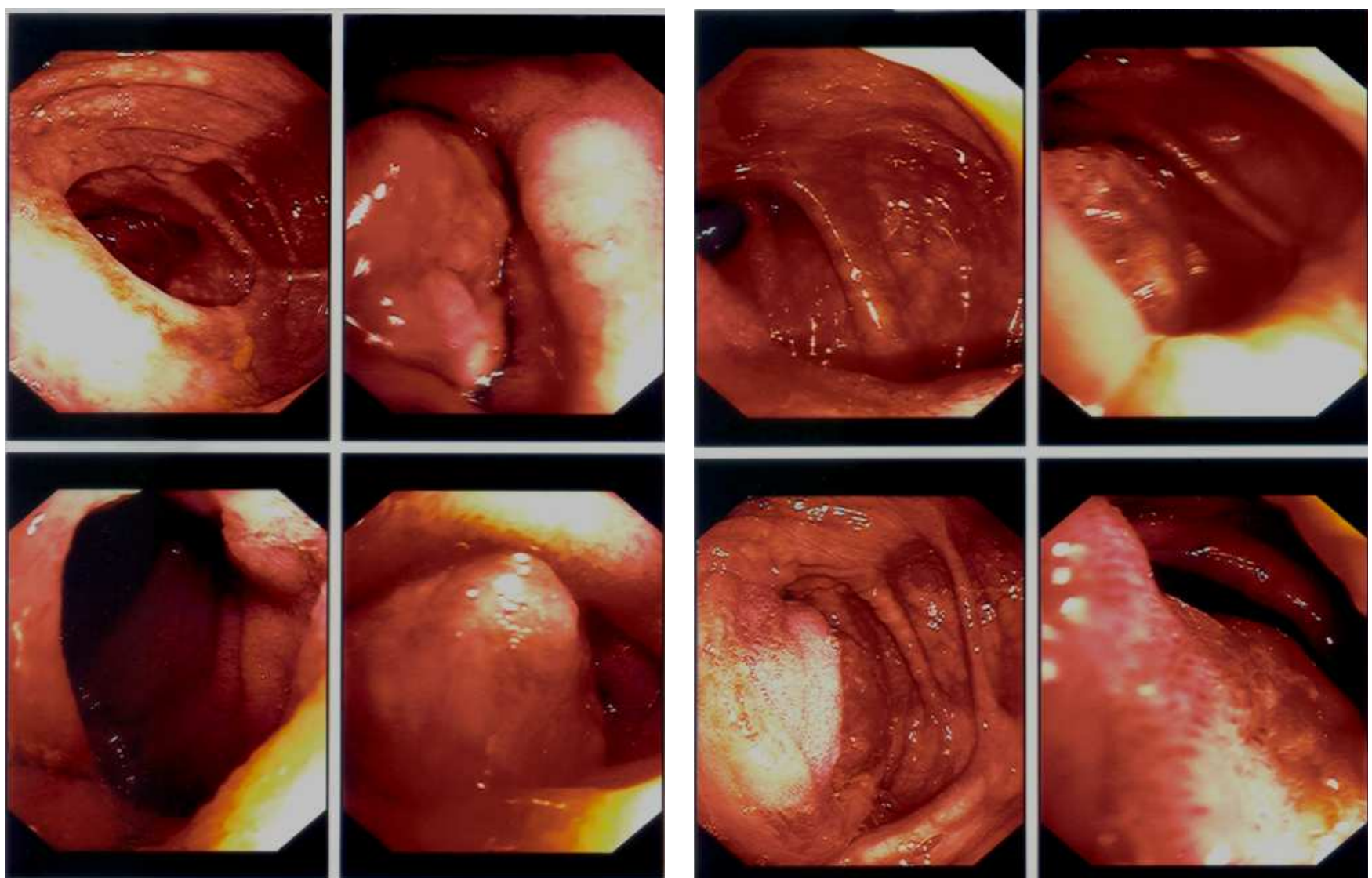

Figura 1 - Fotos da colonoscopia mostrando projeção do íleo terminal através da válvula ileocecal (*) e, após redução lesão nodular de $5 \mathrm{~cm}$ (seta). 
A média de idade de ocorrência de intussuscepções intestinais em adultos é de 50 anos. Os sintomas são inespecíficos, crônicos ou subagudos, relacionados à obstrução parcial intermitente da luz intestinal. Em uma revisão recente de 41 casos, 24,4\% apresentaram um quadro agudo de obstrução intestinal, outros $24,4 \%$ apresentaram quadro subagudo e 51,2\% eram portadores de sintomas crônicos ${ }^{5}$. Os sintomas mais frequentes são cólicas abdominais, associadas a náuseas e vômitos. Diarréia e constipação são sintomas menos freqüentes. Essa sintomatologia e a relativa raridade do quadro fazem do seu diagnóstico um desafio. Séries cirúrgicas mostraram que o diagnostico pré-operatório foi correto em apenas 32 a $50 \%$ dos casos $^{4}$. Como complicações, podemos ter isquemia mesentérica, perfuração, peritonite, choque e até mesmo óbito, caso diagnóstico e tratamento adequados não sejam feitos.

O diagnóstico de certeza pode ser feito com técnicas de imagem não-invasivas. A tomografia computadorizada se mostrou como a melhor ferramenta para o diagnóstico ${ }^{5}$. A ultrassonografia é uma outra modalidade que apresenta alta sensibilidade e, levando-se em conta a realidade de países subdesenvolvidos e a disponibilidade da tomografia em nosso meio, continua sendo o exame de escolha para o diagnóstico. Trabalhos na literatura mostram índice de acurácia de até $100 \%$ para o diagnóstico de intussuscepções na população pediatrica² ${ }^{2}$ Nesse grupo de pacientes, a ultrassonografia tem uma sensibilidade de $97,9 \%$ e especificidade de $97,8 \%$, mostrando que o exame pode ser usado com segurança, desde que exista a suspeita clínica ${ }^{6}$.

As intussuscepções sintomáticas persistentes em adultos devem ser tratadas cirurgicamente devido à alta proporção de casos com lesão estrutural e a alta incidência de malignidade, principalmente no cólon. Não se deve tentar redução da intussuscepção por enema. A maioria dos autores também não recomenda a redução intra-operatória da intussuscepção, a fim de evitar possíveis embolismos venosos do tumor. Intussuscepções de tumores de intestino delgado apresentam menor incidência de malignidade, mas sua ressecção ainda está indicada ${ }^{7}$.

\section{Tumores de Intestino Delgado ${ }^{9-12}$}

Existem aproximadamente 40 tipos histológicos de tumores do intestino delgado. A maioria dos tumores é benigna e eles permanecem assintomáticos, sendo encontrados somente em necropsias, enquanto aqueles que produzem sintomas e são encontrados durante cirurgias se revelam, na maior parte das vezes, malignos.

Os tumores de intestino delgado normalmente se apresentam com queixas não específicas, como dor abdominal em cólica, anemia, hemorragia digestiva ou obstrução intestinal intermitente, que levam o médico a considerar outros diagnósticos diferenciais muito mais comuns. Em uma série de casos publicada em 1996, o tempo médio da primeira queixa ao diagnóstico foi de sete meses. Intussuscepções podem ocorrer, causando obstrução inter- mitente e sangramento. Os lipomas são a causa mais comum dessa complicação ${ }^{4}$

Embora o intestino delgado represente aproximadamente $75 \%$ da extensão do trato gastrointestinal e mais de $90 \%$ da superfície de mucosa, ele raramente desenvolve tumores malignos. Menos de $2 \%$ dos tumores malignos gastrointestinais são originados nessa região ${ }^{5}$. Sua incidência ajustada para idade é de um para cada 100.000 pessoas, sendo sua prevalência de $0,6 \%{ }^{6}$. O motivo pelo qual essa região apresenta uma carcinogênese tão pequena se comparada com o estômago e cólon, regiões vizinhas de alto risco para neoplasias, permanece obscura, embora várias hipóteses tenham sido postuladas.

Os leiomiomas são responsáveis por quase um quarto dos tumores benignos de intestino delgado, sendo o representante mais comum desse grupo e aquele que produz manifestações clínicas com maior freqüência. Os adenomas são o segundo maior representante desse grupo, e são os tumores benignos assintomáticos mais comuns. Em um estudo retrospectivo de 192 adenomas vilosos do duodeno, 42\% apresentaram transformação maligna?. Devido a esse risco de malignização, esses tumores devem ser removidos sempre, preferencialmente por via endoscópica. Os lipomas são o terceiro tumor benigno mais importante no intestino delgado, e mais de dois terços deles permanece assintomático. Outros representantes mais raros desse grupo de tumores são os hamartomas e hemangiomas.

Já os tumores malignos têm como principal representante o adenocarcinoma (30 a 50\%), mais comuns no duodeno proximal e jejuno, com pico de incidência na sétima década de vida e maior acometimento de homens. A ampola de Vaters é o sítio mais comum de adenocarcinomas de intestino delgado. O principal fator de risco é a presença de adenoma, único ou múltiplo. A apresentação mais comum dos adenocarcinomas é obstrução intestinal, sangramento, perda de peso e icterícia. Eles se tornam sintomáticos mais precocemente que outros tumores, permitindo diagnóstico e intervenção terapêutica mais precoce. Apesar disso, os carcinomas de intestino delgado já apresentam metástases no momento do diagnóstico. Os linfomas representam 15 a 20\% dos tumores primários malignos do intestino delgado e são a segunda causa mais comum destes. O trato gastrointestinal é o sítio extranodal mais comum de apresentação de linfomas, sendo responsável por 5 a $20 \%$ dos linfomas não-Hodgkin. Leiomissarcomas são a terceira entidade mais comum entre os tumores malignos do intestino delgado, acometendo principalmente o jejuno, com pico de incidência na sexta década de vida.

\section{Linfoma de Burkitt ${ }^{13}$}

Descrito pela primeira vez por Denis Burkitt em 1958, o Linfoma de Burkitt é um linfoma não-Hodgkin altamente agressivo que se apresenta freqüentemente em sítios extranodais ou como leucemia aguda. O marcador dessa doença é a superexpressão de c-Myc, resultando de uma translocação do gene $c-m y c$ do cromossomo 8 no 
cromossomo 14 (80\% dos casos). A alteração da expressão do C-Myc, um importante fator de transcrição influencia a fabricação de diversas proteínas envolvidas na regulação do ciclo celular, apoptose, crescimento celular, adesão celular e diferenciação. As células do linfoma de Burkitt expressam em sua superfície IgM, Bcl-6, CD19, CD20, CD22, CD10 e CD79a, e são negativas para CD5, CD23 e Tdt.

Três variantes clínicas do linfoma de Burkitt foram descritas: endêmica, esporádica e associado à imunodeficiência. A primeira acomete principalmente crianças de quatro a sete anos na África equatorial, com envolvimento frequente da mandíbula e rins e sua designação se deve a sua distribuição semelhante a da malária. 0 linfoma de Burkitt associado à imunodeficiência é observado em pacientes infectados pelo vírus da imunodeficiência humana, mas ao contrário dos outros linfomas associado ao HIV, é observado em pacientes com contagem de CD4 superior a 200 células/ $\mu \mathrm{L}$. A variante dita esporádica é responsável por 1 a $2 \%$ dos linfomas em adultos na Europa ocidental e EUA, e se apresenta principalmente como tumores abdominais, sem distribuição climática ou geográfica específica.
Sintomas podem incluir dor abdominal, náuseas, vômitos, obstrução intestinal, sangramento gastrointestinal ou síndromes que mimetizam apendicite aguda ou intussuscepções. As apresentações abdominais normalmente afetam o intestino ou linfonodos intra-abdominais, embora outros órgãos podem ser afetados. Em adultos, acometimento da medula óssea foi relatado em 30 a 38\% dos casos, enquanto o sistema nervoso central foi atingido em 13 a $17 \%$ dos casos.

Os linfomas de Burkitt apresentam fração de crescimento elevada, dobrando de tamanho a cada 25 horas. Novas abordagens quimioterápicas de curta duração, com regimes intensivos que minimizam os intervalos entre as aplicações e mantêm concentração das drogas por pelo menos 48 a 72 horas apresentam grande eficácia no tratamento da doença. Vários estudos sugerem que esses tratamentos podem ser curativos, mesmo em doença de estágio avançado. Dependendo do estudo, 65 a 100\% dos adultos obtiveram remissão completa do quadro, com manutenção de 47 a $86 \%$ dos resultados no ano seguinte ao tratamento. Mielossupressão e infecções foram as complicações mais frequentemente encontradas ${ }^{5}$.

\title{
A B S T R A C T
}

\begin{abstract}
Although intussusception is the main cause of intestinal obstruction in the pediatric population, it is a rare condition in adults, and when it happens, the clinical characteristics differ a lot from the pediatric group. The purpose of this article is to report a case of a 16 years-old male that was seen at the Emergency Room of the University Hospital of São Paulo with an intestinal intussusception as the first clinical presentation of a Burkitt lymphoma. A literature review was carried out and relevant aspects of the case are discussed.
\end{abstract}

Key words: Intussusceptions. Abdomen, Acute. Lymphoma. Burkitt lymphoma.

\section{REFERENCIAS}

1. Brayton D, Norris WJ. Intussusception in adults. Am J Surg. 1954; 88(1):32-43

2. Lehnert $T$, Sorge $T$, Till $H$, Rolle $U$. Intussusception in childrenclinical presentation, diagnosis and management. Int J Colorectal Dis. 2009; 24(10):1187-92. Epub 2009 May 6.

3. Hanan $B$, Diniz TR, da Luz MM, da Conceição $S A$, da Silva RG, Lacerda-Filho A. Intussusception in adults. Colorectal Dis. 2009 Apr 10. [Epub ahead of print].

4. Huang BY, Warshauer DM. Adult intussusception: diagnosis and clinical relevance. Radiol Clin North Am. 2003; 41(6):1137-51.

5. Wang N, Cui XY, Liu Y, LongJ, Xu YH, Guo RX, Guo KJ. Adult intussusception: a retrospective review of 41 cases. World J Gastroenterol. 2009; 15(26):3303-8.

6. Hryhorczuk AL, Strouse PJ. Validation of US as a first-line diagnostic test for assessment of pediatric ileocolic intussusception. Pediatr Radiol. 2009; 39(10):1075-9. Epub 2009 Aug 6.

7. Yakan S, Caliskan C, Makay O, Denecli AG, Korkut MA. Intussusception in adults: clinical characteristics, diagnosis and operative strategies. World J Gastroenterol. 2009; 15(16):1985-9.

8. Daneman A, Navarro O. Intussusception. Part 1: a review of diagnostic approaches. Pediatr Radiol. 2003; 33(2):79-85. Epub 2002 Nov 19
9. Gill SS, Heuman DM, Mihas AA. Small intestinal neoplasms. J Clin Gastroenterol. 2001; 33(4):267-82.

10. Barclay TH, Schapira DV. Malignant tumors of the small intestine. Cancer. 1983; 51(5):878-81.

11. Attanoos R, Williams GT. Epithelial and neuroendocrine tumors of the duodenum. Semin Diagn Pathol. 1991; 8(3):149-62.

12. Witteman BJ, Janssens AR, Griffioen G, Lamers CB. Villous tumours of the duodenum. An analysis of the literature with emphasis on malignant transformation. Neth J Med. 1993; 42(1-2):5-11.

13. Blum KA, Lozanski G, Byrd JC. Adult Burkitt leukemia and lymphoma. Blood. 2004; 104(10):3009-20. Epub 2004 Jul 20.

Recebido em 16/05/2006

Aceito para publicação em 11/07/2006

Conflito de interesse: nenhum

Fonte de financiamento: nenhuma

\section{Como citar este artigo:}

Batista BN, Maximiano LF. Intussuscepção intestinal em adultos jovens - revisão de literatura. Rev Col Bras Cir. [periódico na Internet] 2009; 36(6). Disponível em URL: http://www.scielo.br/rcbc

\section{Endereço para correspondência:}

Bernardo Nogueira Batista

E-mail: bernardonb@uol.com.br 